\title{
Relationships between initial unloading slope, contact depth, and mechanical properties for spherical indentation in linear viscoelastic solids
}

\author{
Yang-Tse Cheng ${ }^{\mathrm{a}, *}$, Che-Min Cheng ${ }^{\mathrm{b}}$ \\ a Materials and Processes Laboratory, MS: 480-106-224, General Motors Research and Development Center, \\ 30500 Mount Road, Warren, MI 48090, USA \\ ${ }^{\mathrm{b}}$ Institute of Mechanics, Chinese Academy of Sciences, Beijing 100080, China \\ Received in revised form 11 May 2005; accepted 24 May 2005
}

\begin{abstract}
Using analytical and finite element modeling, we examine the relationships between initial unloading slope, contact depth, and mechanical properties for spherical indentation in viscoelastic solids with either displacement or load as the independent variable. We then investigate whether the Oliver-Pharr method for determining the contact depth and contact radius, originally proposed for indentation in elastic and elastic-plastic solids, is applicable to spherical indentation in viscoelastic solids. Finally, the analytical and numerical results are used to answer questions raised in recent literature about measuring viscoelastic properties from instrumented spherical indentation experiments.
\end{abstract}

(C) 2005 Elsevier B.V. All rights reserved.

Keywords: Indentation; Viscoelastic; Mechanical properties; Contact mechanics; Modeling; Finite element

\section{Introduction}

Instrumented indentation is playing an increasing role in the study of small-scale mechanical behavior of "soft" matters, such as polymers, composites, biomaterials, and food products. Since many of these materials exhibit viscoelastic behavior, it is important to have a sound understanding of instrumented indentation in viscoelastic solids. Although instrumented indentation has recently become popular for measuring the small scale mechanical properties of soft materials, theoretical studies of linear viscoelastic bodies in contact became active since the mid 1950s by the work of Lee [1], Radok [2], Lee and Radok [3], Hunter [4], Graham [5,6], Yang [7], and Ting [8,9]. In recent years, a number of authors have extended the early work to the analysis of indentation measurements in viscoelastic solids using either conical or spherical indenters [10-15]. It has been reported, however, that a most commonly used method due to Oliver and Pharr [16,17] for analyzing instrumented indentation experiments does not produce accurate results when it is applied to

\footnotetext{
* Corresponding author. Tel.: +1 586986 0939; fax: +1 5869863091 .

E-mail address: yang.t.cheng@gm.com (Y.-T. Cheng).
}

spherical indentation in viscoelastic solids $[18,19]$. In this paper, we investigate spherical indentation in linear viscoelastic solids using analytical and finite element modeling. Specifically, we examine the relationship between initial unloading slope, contact depth, and mechanical properties for spherical indentation in linear viscoelastic solids since this relationship is the basis for the Oliver-Pharr method. We then investigate whether the commonly used Oliver-Pharr method for determining the contact depth or contact area is applicable to spherical indentation in viscoelastic solids. This work, together with our two previous publications on conical indentation in linear viscoelastic solids [20,21], helps improve the understanding of indentation in linear viscoelastic solids and resolve questions raised in recent literature about measuring viscoelastic properties from spherical, as well as conical indentation experiments.

\section{Analytical results}

\subsection{Spherical indentation in linear viscoelastic solids}

We consider a rigid, smooth, and frictionless spherical indenter with a radius $R$ indenting a viscoelastic solid that can be 
described by the following constitutive relationships [22,23] between deviatoric stress and strain, $s_{i j}$ and $d_{i j}$, and between dilatational stress and strain, $\sigma_{i i}$ and $\varepsilon_{i i}$,

$s_{i j}(t)=2 \int_{0}^{t} G(t-\tau) \frac{\partial d_{i j}(\tau)}{\partial \tau} \mathrm{d} \tau$
$\sigma_{i i}(t)=3 \int_{0}^{t} K(t-\tau) \frac{\partial \varepsilon_{i i}(\tau)}{\partial \tau} \mathrm{d} \tau$

where $G(t)$ is the relaxation modulus in shear and $K(t)$ is the relaxation modulus in dilatation. The time-dependent Young's modulus and Poisson's ratio are then given by $E(t)=[9 K(t) G(t)] /[3 K(t)+G(t)]$ and $\nu(t)=[E(t) / 2 G(t)]-1$, respectively.

Alternatively, the stress-strain relations can be written as $[22,23]$

$2 d_{i j}(t)=\int_{0}^{t} J_{1}(t-\tau) \frac{\partial s_{i j}(\tau)}{\partial \tau} \mathrm{d} \tau$
$3 \varepsilon_{i i}(t)=\int_{0}^{t} J_{2}(t-\tau) \frac{\partial \sigma_{i i}(\tau)}{\partial \tau} \mathrm{d} \tau$

where $J_{1}(t)$ is the shear compliance and $J_{2}(t)$ is the volumetric compliance. Obviously, Eqs. (1) and (2) are not independent of each other. In fact, the relationships between the relaxation and creep functions, $G(t)$ and $J_{1}(t)$, as well as $K(t)$ and $J_{2}(t)$, have simple forms after transforming them using Laplace transformation $[22,23]$,

$\int_{0}^{t} J_{1}(t-\tau) G(\tau) \mathrm{d} \tau=\int_{0}^{t} J_{1}(\tau) G(t-\tau) \mathrm{d} \tau=t$
$\int_{0}^{t} J_{2}(t-\tau) K(\tau) \mathrm{d} \tau=\int_{0}^{t} J_{2}(\tau) K(t-\tau) \mathrm{d} \tau=t$

As a consequence, we have,

$$
\begin{aligned}
& J_{1}(0) G(0)=J_{1}(\infty) G(\infty)=1 \\
& J_{2}(0) K(0)=J_{2}(\infty) K(\infty)=1
\end{aligned} .
$$

In the following, we assume that Poisson's ratio is time independent. Consequently, either $G(t)$ and $v$ or $J_{1}(t)$ and $v$ are sufficient to describe the linear viscoelastic behavior.

\subsection{Relationships between load, displacement, and contact depth}

In the "classical spherical" indenter approximation, where the indenter shape is a paraboloid of revolution, the relationship between force, $F(t)$, and displacement, $h(t)$, is given by [1-9]:

$F(t)=\frac{8 \sqrt{R}}{3(1-v)} \int_{0}^{t} G(t-\tau) \frac{\mathrm{d} h^{3 / 2}(\tau)}{\mathrm{d} \tau} \mathrm{d} \tau$, when the displacement is the independent variable. When the force is the independent variable, the relationship between displacement, $h(t)$, and force, $F(t)$, is given by [1-9]:

$h^{3 / 2}(t)=\frac{3(1-\nu)}{8 \sqrt{R}} \int_{0}^{t} J_{1}(t-\tau) \frac{\mathrm{d} F(\tau)}{\mathrm{d} \tau} \mathrm{d} \tau$.

The load-displacement relationship can therefore be obtained if the viscoelastic properties of materials, $G(t)$ or $J_{1}(t)$, and $v$, are known using the respective Eq. (5) or (6). Conversely, the viscoelastic properties may be obtained from measured $F(t)$ versus $h(t)$ relations by solving integral Eq. (5) or (6) [10-15]. Eqs. (5) and (6) reduce to the well-known equation for spherical indentation into purely elastic solids [24],

$F=\frac{8}{3} \frac{G}{1-v} \sqrt{R} h^{3 / 2}$,

where $G=1 / J_{1}$ and $v$ are the time-independent shear modulus and Poisson's ratio, respectively.

Eqs. (5) and (6) are special cases of more general expressions derived by Graham [5] and Ting [8]. They showed that Eqs. (5) and (6) are valid when the contact area is a monotonically increasing function of time. Under the same condition, the relationship between contact depth, $h_{\mathrm{c}}(t)$, and contact radius, $a(t)$, and indenter displacement, $h(t)$, is the same as that in the purely elastic case [5,8],

$h(t)=\frac{a^{2}(t)}{R}=2 h_{\mathrm{c}}(t)$

The equations for unloading where the contact area decreases monotonically have also been derived [5,8], though they are more complicated. As a result, a number of authors have proposed methods for deducing $G(t)$ or $J_{1}(t)$ from indentation loading curves using Eq. (5) or (6) without using the information contained in the indentation unloading curves [18,25]. However, Eqs. (5) and (8) may be used to evaluate the initial unloading slope of unloading curves, as we have shown in the similar case of conical indentation in linear viscoelastic solids [20,21]. In Section 2.2, we derive equations for initial unloading slopes using Eqs. (5), (6), and (8). In Section 3, we validate the initial unloading slope equations using finite element calculations and discuss the applicability of the Oliver-Pharr method for determining contact depth or contact area.

\subsection{Relationships between initial unloading slope, contact depth, and viscoelastic property}

\subsubsection{Initial unloading slope when displacement is the independent variable}

Suppose unloading takes place at $t=t_{\mathrm{m}}$ with a constant unloading rate of $\left.\frac{\mathrm{d} h}{\mathrm{~d} t}\right|_{t_{\mathrm{m}}^{+}}=-v_{\mathrm{h}}$, we have, using Eq. (5) for 
$0 \leq t \leq t_{\mathrm{m}}$ and $\Delta t \rightarrow 0$

$$
\begin{aligned}
\frac{F\left(t_{\mathrm{m}}+\Delta t\right)-F\left(t_{\mathrm{m}}\right)}{\Delta t}= & \frac{8 \sqrt{R}}{3(1-v) \Delta t}\left[\int_{0}^{t_{\mathrm{m}}+\Delta t} G\left(t_{\mathrm{m}}+\Delta t-\tau\right) \frac{\mathrm{d} h^{3 / 2}(\tau)}{\mathrm{d} \tau} \mathrm{d} \tau-\int_{0}^{t_{\mathrm{m}}} G\left(t_{\mathrm{m}}-\tau\right) \frac{\mathrm{d} h^{3 / 2}(\tau)}{\mathrm{d} \tau} \mathrm{d} \tau\right] \\
= & \frac{8 \sqrt{R}}{3(1-v) \Delta t}\left[\int_{0}^{t_{\mathrm{m}}} G\left(t_{\mathrm{m}}+\Delta t-\tau\right) \frac{\mathrm{d} h^{3 / 2}(\tau)}{\mathrm{d} \tau} \mathrm{d} \tau+\int_{t_{\mathrm{m}}}^{t_{\mathrm{m}}+\Delta t} G\left(t_{\mathrm{m}}+\Delta t-\tau\right) \frac{\mathrm{d} h^{3 / 2}(\tau)}{\mathrm{d} \tau} \mathrm{d} \tau\right. \\
& \left.-\int_{0}^{t_{\mathrm{m}}} G\left(t_{\mathrm{m}}-\tau\right) \frac{\mathrm{d} h^{3 / 2}(\tau)}{\mathrm{d} \tau} \mathrm{d} \tau\right] \\
= & \frac{8 \sqrt{R}}{3(1-v) \Delta t}\left[\int_{0}^{t_{\mathrm{m}}} G\left(t_{\mathrm{m}}-\tau\right) \frac{\mathrm{d} h^{3 / 2}(\tau)}{\mathrm{d} \tau} \mathrm{d} \tau+\left.\Delta t \int_{0}^{t_{\mathrm{m}}} \frac{\mathrm{d} G}{\mathrm{~d} \eta}\right|_{\eta=t_{\mathrm{m}}-\tau} \frac{\mathrm{d} h^{3 / 2}(\tau)}{\mathrm{d} \tau} \mathrm{d} \tau\right. \\
& \left.+G(0) \frac{\mathrm{d} h^{3 / 2}\left(t_{\mathrm{m}}^{+}\right)}{\mathrm{d} t} \Delta t-\int_{0}^{t_{\mathrm{m}}} G\left(t_{\mathrm{m}}-\tau\right) \frac{\mathrm{d} h^{3 / 2}(\tau)}{\mathrm{d} \tau} \mathrm{d} \tau\right] \\
= & \frac{8 \sqrt{R}}{3(1-v)}\left[\left.\int_{0}^{t_{\mathrm{m}}} \frac{\mathrm{d} G}{\mathrm{~d} \eta}\right|_{\eta=t_{\mathrm{m}}-\tau} \frac{\mathrm{d} h^{3 / 2}(\tau)}{\mathrm{d} \tau} \mathrm{d} \tau-\frac{3}{2} G(0) h^{1 / 2}\left(t_{\mathrm{m}}^{+}\right) v_{\mathrm{h}}\right]
\end{aligned}
$$

The initial unloading slope is then given by, using $\frac{\mathrm{d} F}{\mathrm{~d} h}=\frac{\mathrm{d} F / \mathrm{d} t}{\mathrm{~d} h / \mathrm{d} t}$ and Eq. (8),

$$
\begin{gathered}
\frac{\mathrm{d} F}{\mathrm{~d} h}=\frac{8 \sqrt{R}}{3(1-v)}\left[\frac{3}{2} G(0) h^{1 / 2}\left(t_{\mathrm{m}}^{+}\right)-\left.\frac{1}{v_{\mathrm{h}}} \int_{0}^{t_{\mathrm{m}}} \frac{\mathrm{d} G}{\mathrm{~d} \eta}\right|_{\eta=t_{\mathrm{m}}-\tau}\right. \\
\left.\frac{\mathrm{d} h^{3 / 2}(\tau)}{\mathrm{d} \tau} \mathrm{d} \tau\right] \stackrel{v_{\mathrm{h}} \rightarrow \infty}{\approx} \frac{4 \sqrt{R h}}{1-v} G(0)=\frac{4 G(0) a}{1-v}
\end{gathered}
$$

\subsubsection{Initial unloading slope when force is the independent} variable

Suppose unloading takes place at $t=t_{\mathrm{m}}$ with a constant unloading rate of $\left.\frac{\mathrm{d} F(t)}{\mathrm{d} t}\right|_{t_{\mathrm{m}}^{+}}=-v_{\mathrm{F}}$, we have, using Eq. (6) for $0 \leq t \leq t_{\mathrm{m}}+\Delta t$ and $\Delta t \rightarrow 0$,

$$
\begin{aligned}
\frac{h^{3 / 2}\left(t_{\mathrm{m}}+\Delta t\right)-h^{3 / 2}\left(t_{\mathrm{m}}\right)}{\Delta t}= & \frac{3(1-v)}{8 \sqrt{R} \Delta t}\left[\int_{0}^{t_{\mathrm{m}}+\Delta t} J_{1}\left(t_{\mathrm{m}}+\Delta t-\tau\right) \frac{\mathrm{d} F(\tau)}{\mathrm{d} \tau} \mathrm{d} \tau-\int_{0}^{t_{\mathrm{m}}} J_{1}\left(t_{\mathrm{m}}-\tau\right) \frac{\mathrm{d} F(\tau)}{\mathrm{d} \tau} \mathrm{d} \tau\right] \\
= & \frac{3(1-v)}{8 \sqrt{R} \Delta t}\left[\int_{0}^{t_{\mathrm{m}}} J_{1}\left(t_{\mathrm{m}}-\tau\right) \frac{\mathrm{d} F(\tau)}{\mathrm{d} \tau} \mathrm{d} \tau+\left.\Delta t \int_{0}^{t_{\mathrm{m}}} \frac{\mathrm{d} J_{1}(\eta)}{\mathrm{d} \eta}\right|_{\eta=t_{\mathrm{m}}-\tau} \frac{\mathrm{d} F(\tau)}{\mathrm{d} \tau} \mathrm{d} \tau\right. \\
& \left.+J_{1}(0) \frac{\mathrm{d} F\left(t_{\mathrm{m}}^{+}\right)}{\mathrm{d} t} \Delta t-\int_{0}^{t_{\mathrm{m}}} J_{1}\left(t_{\mathrm{m}}-\tau\right) \frac{\mathrm{d} F(\tau)}{\mathrm{d} \tau} \mathrm{d} \tau\right] \\
= & \frac{3(1-v)}{8 \sqrt{R}}\left[\left.\int_{0}^{t_{\mathrm{m}}} \frac{\mathrm{d} J_{1}(\eta)}{\mathrm{d} \eta}\right|_{\eta=t_{\mathrm{m}}-\tau} \frac{\mathrm{d} F(\tau)}{\mathrm{d} \tau} \mathrm{d} \tau-J_{1}(0) v_{\mathrm{F}}\right]
\end{aligned}
$$

Eqs. (10) and (12) suggest that the initial unloading slopes converge when the unloading rate, either in displacement or load case, i.e.

$\left.\frac{\mathrm{d} F}{\mathrm{~d} h}\right|_{h=h_{\mathrm{m}}}=\frac{4 G(0) a}{1-v}$,

since $G(0)=1 / J(0)$ according to Eq. (4). Thus, the "instantaneous" moduli, $G(0) /(1-v)$ or $E(0) /\left(1-v^{2}\right)$, can be obtained provided that the contact radius, $a$, or contact depth, $h_{\mathrm{c}}$, is known as a function of $h_{\mathrm{m}}=h\left(t_{\mathrm{m}}\right)$. The latter condition is provided by Eq. (8). An interesting, though not necessarily surprising, implication of Eqs. (10) and (12) is that, aside from the requirement that the contact area should remain monotonically control, is sufficiently fast. Once this limiting case is reached, Eqs. (10) and (12) become the same as that for purely elastic
The initial unloading slope is then given by, using $\frac{\mathrm{d} F}{\mathrm{~d} h}=\frac{\mathrm{d} F / \mathrm{d} t}{\mathrm{~d} h / \mathrm{d} t}=$ $-\frac{\nu_{\mathrm{F}}}{\mathrm{d} h / \mathrm{d} t}=\frac{-(3 / 2) h^{1 / 2} \nu_{\mathrm{F}}}{\mathrm{d} h^{3 / 2} / \mathrm{d} t}$ and Eq. (8),

$$
\begin{aligned}
\frac{\mathrm{d} F}{\mathrm{~d} h}= & -\frac{\frac{3}{2} h^{1 / 2} v_{\mathrm{F}}}{\frac{\mathrm{d} h^{3 / 2}}{\mathrm{~d} t}} \\
= & \frac{4 \sqrt{R h}}{1-v} \frac{1}{J(0)-\left.\frac{1}{v_{\mathrm{F}}} \int_{0}^{t_{\mathrm{m}}} \frac{\mathrm{d} J(\eta)}{\mathrm{d} \eta}\right|_{\eta=t_{\mathrm{m}}-\tau} \frac{\mathrm{d} F(\tau)}{\mathrm{d} \tau} \mathrm{d} \tau} \\
& v_{\mathrm{F} \rightarrow \infty} \underset{\frac{4 a}{1-v} \frac{1}{J(0)} .}{ }
\end{aligned}
$$


Based on the results of Sneddon [24], Oliver and Pharr developed an expression for, $h_{\mathrm{c}}$, at the indenter displacement, $h_{\mathrm{c}}$,

$h_{\mathrm{c}}=h_{\mathrm{m}}-\xi \frac{F_{\mathrm{m}}}{(\mathrm{d} F / \mathrm{d} h)_{\mathrm{m}}}$,

where $F_{\mathrm{m}}$ and $(\mathrm{d} F / \mathrm{d} h)_{\mathrm{m}}$ are the respective load and the initial slope of the unloading curve at the indenter displacement depth, $h_{\mathrm{m}}$. The numerical value of $\xi$ is $3 / 4$ for paraboloids of revolution ("classical spherical" indenter). Although Eq. (14) was derived from solutions to elastic contact problems, it has been used to estimate contact depth for indentation in elastic-plastic solids [16,17] and viscoelastic solids [18,19,25,26,29-34]. In the following, we examine the conditions for using Eq. (13) and the applicability of Eq. (14) by analyzing the complete loading-unloading curves and contact depths using finite element calculations.

\section{Numerical results}

\subsection{Finite element model}

We consider a frictionless, spherical indenter of radius $R=2 \mu \mathrm{m}$ indenting an isotropic, three-parameter "standard" linear viscoelastic model solid (see Fig. 1). The shear and hydrostatic relaxation modulus are given by:

$$
\begin{aligned}
& G(t)=G_{1}\left(1-\frac{G_{1}}{G_{1}+G_{2}}\left(1-\mathrm{e}^{-t / \tau_{s}}\right)\right), \\
& K(t)=K_{1}\left(1-\frac{K_{1}}{K_{1}+K_{2}}\left(1-\mathrm{e}^{-t / \tau_{s}}\right)\right)
\end{aligned}
$$

where the relaxation time $\tau_{\mathrm{s}}=\eta /\left(G_{1}+G_{2}\right)$. Various materials parameters are given as $G_{1}=235 \mathrm{MPa}, G_{2}=25.8 \mathrm{MPa}$, $K_{1}=688 \mathrm{MPa}, K_{2}=75.6 \mathrm{MPa}$, and $t=0.99 \mathrm{~s}$. The parameters are chosen such that Poisson's ratio, $v=0.483$, is time independent, though both $G(t)$ and $K(t)$ are time dependent. Specifically, their "instantaneous" and "equilibrium" values at the respective $t=0$ and $\infty$ are as follows: $G(0)=235 \mathrm{MPa}$ and $G(\infty)=23.2 \mathrm{MPa}$; $K(0)=688 \mathrm{MPa}$ and $K(\infty)=68.1 \mathrm{MPa}$. The parameters of this fictitious model solid are used for illustration purposes. Because of linearity, the results can be scaled to represent other materials of the same general type when the dimensionless parameters, such as $G_{1} / G_{2}, K_{1} / K_{2}, G_{1} / K_{1}$, and $t / \tau_{\mathrm{s}}$, are equal. It is important to note that $G(0) / G(\infty)$ is about 10 which is typical of many polymers and biomaterials. Finite element calculations were carried out using the classical isotropic linear viscoelastic model implemented in ABAQUS [27] using either displacement or load as

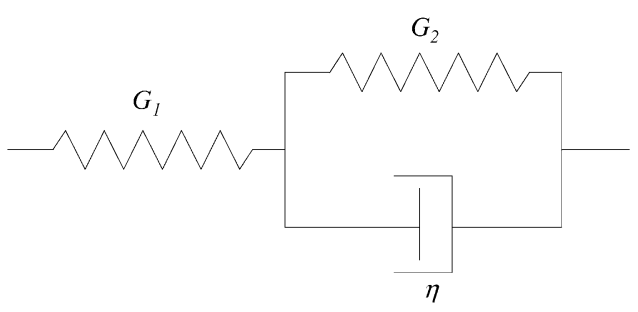

Fig. 1. A three-parameter "standard" model for linear viscoelastic solids.
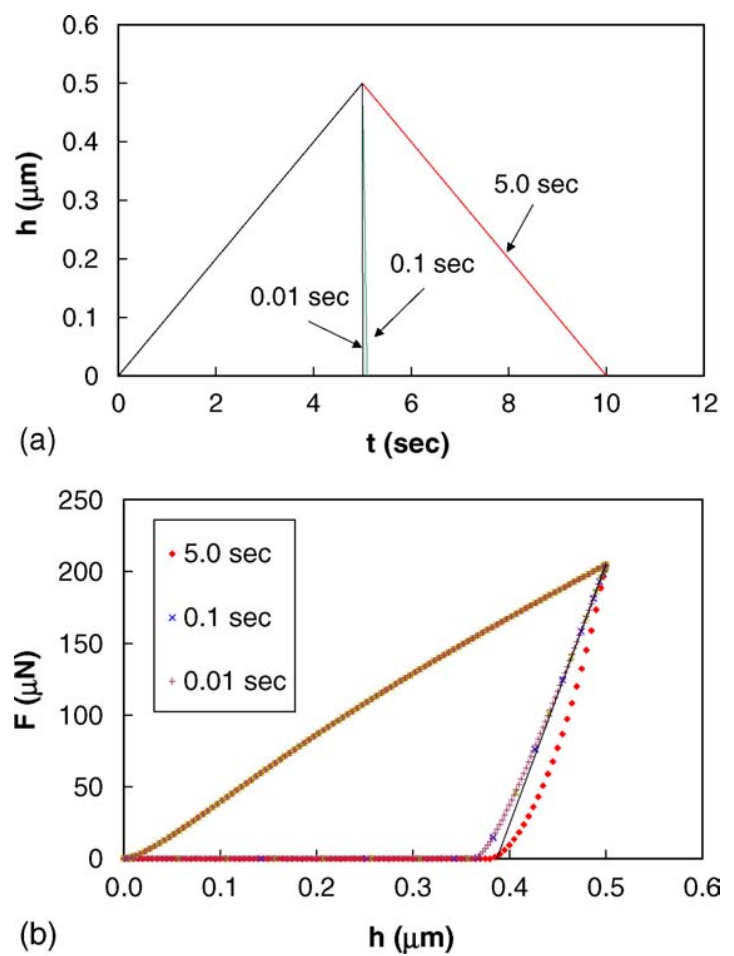

Fig. 2. Displacement-time profiles (a) and the calculated loading-unloading curves (b) for the same loading rate and three different unloading rates. The tangent line with initial unloading slope is also shown for the converged unloading curve (b). The loading-unloading curves are labeled by the time duration of unloading.

the independent variable. The finite element mesh is the same as that used in Ref. [28].

\subsection{Displacement control}

For constant indentation displacement rate profiles given in Fig. 2a, the corresponding loading-unloading curves from finite element calculations are shown in Fig. 2b. These examples clearly show that, for the same loading history, the initial unloading slopes converge when unloading rate is sufficiently fast, in agreement with Eq. (10). A tangent line with the converged initial unloading slope is also shown in Fig. 2b. Furthermore, Fig. $2 \mathrm{~b}$ suggests that the complete unloading curve converges to one limiting case as the unloading rate increases.

\subsection{Load control}

For constant indentation loading rate profiles given in Fig. 3a, the corresponding loading-unloading curves from finite element calculations are shown in Fig. 3b. When unloading is slow, the indentation depth continues to increase after the force reaches a maximum, resulting in a "bulge" or "nose" in the unloading curve, which has also been observed experimentally $[25,26,29-34]$. This bulge is the consequence of material memory effect carried over from the continuing forward movement of the indenter prior to unloading. Because of this forward movement of the indenter, the maximum contact area occurs after the force maximum. This delayed maximum contact area behavior 

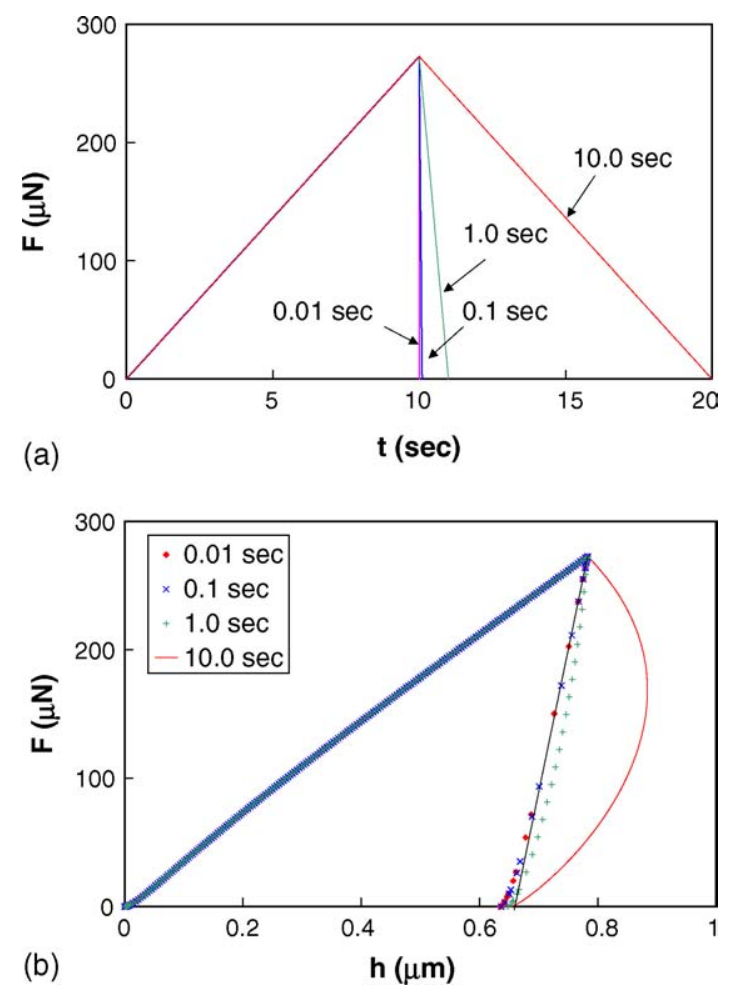

Fig. 3. Force-time profiles (a) and the calculated loading-unloading curves (b) for the same loading rate and four different unloading rates. The tangent line with initial unloading slope is also shown for the converged unloading curve (b). The loading-unloading curves are labeled by the time duration of unloading.

was predicted by the analytical theories of Graham [5] and Ting $[8,9]$, and was verified for conical indentation by numerical calculations $[20,21]$. With increasing unloading rate, the "bulge" disappears. For the same loading history, the initial unloading slope converges when the unloading rate is sufficiently fast, in agreement with Eq. (12). Furthermore, the entire unloading curve converges to one limiting case as the unloading rate increases.

\subsection{Contact depth and unloading slope}

The contact area, $A$, and contact depth, $h_{\mathrm{c}}$, are also obtained from finite element calculations. The calculations show $h_{\mathrm{c}} / h \approx 0.52 \pm 0.01$, which is slightly larger than $1 / 2$ predicted by Eq. (8). This suggests that Eq. (8) needs to be slightly modified to become,

$\frac{h_{\mathrm{c}}}{h}=\alpha \frac{1}{2}$,

where $\alpha=1.04 \pm 0.02$. Furthermore, the finite element results show that there is a small correction to Eq. (10),

$$
\left.\frac{\mathrm{d} F}{\mathrm{~d} h}\right|_{h=h_{\mathrm{m}}}=\beta \frac{4 G(0) a}{1-v}=\beta \frac{2}{\sqrt{\pi}} \frac{E(0)}{1-v^{2}} \sqrt{A},
$$

where $\beta=1.02 \pm 0.01$. The same $\beta$ correction factor has also been seen in the modeling of conical indentation in purely elastic solids and in elastic-plastic solids $[17,20]$. The origin of this correction factor has been discussed previously $[17,20]$. It has been attributed to the fact that Eq. (10) was derived using linearized boundary conditions and infinitesimal theory of continuum mechanics, finite element calculations take into account non-linear effects, including large strain and moving contact boundaries [20]. Because the values of $\alpha$ and $\beta$ are nearly the same as that for purely elastic and elastic-plastic cases, we believe they are insensitive to the particular choice of viscoelastic properties used in the finite element calculations. Although further improvement in the precision and accuracy of the $\alpha$ and $\beta$ values is possible by additional calculations, either Eqs. (16) and (17) or (8) and (13) can be used to obtain, to within 5\% of accuracy, contact depth, $h_{\mathrm{c}}$, and "instantaneous" moduli, $G(0) /(1-v)$ or $E(0) /\left(1-v^{2}\right)$, when unloading rate is sufficiently fast.

\subsection{Oliver-Pharr method}

We now discuss the applicability of the Oliver-Pharr method for estimating contact depth for indentation in viscoelastic solids. Finite element calculations were carried out using constant indentation displacement rate profiles given in Fig. 4a. The load-displacement curves in Fig. $4 \mathrm{~b}$ show that the force required to reach a given indentation depth increases with the loading rate, consistent with the expected behavior of viscoelastic solids. The unloading rates chosen in the calculations are sufficiently fast so that they generate the corresponding converged unloading curves. Furthermore, finite element calculations show that the contact depth, $h_{\mathrm{c}}$, is the same for all three cases shown in Fig. $5 \mathrm{~b}$, as expected from Eq. (8) or (16) since $h_{\mathrm{m}}$ is the same. According
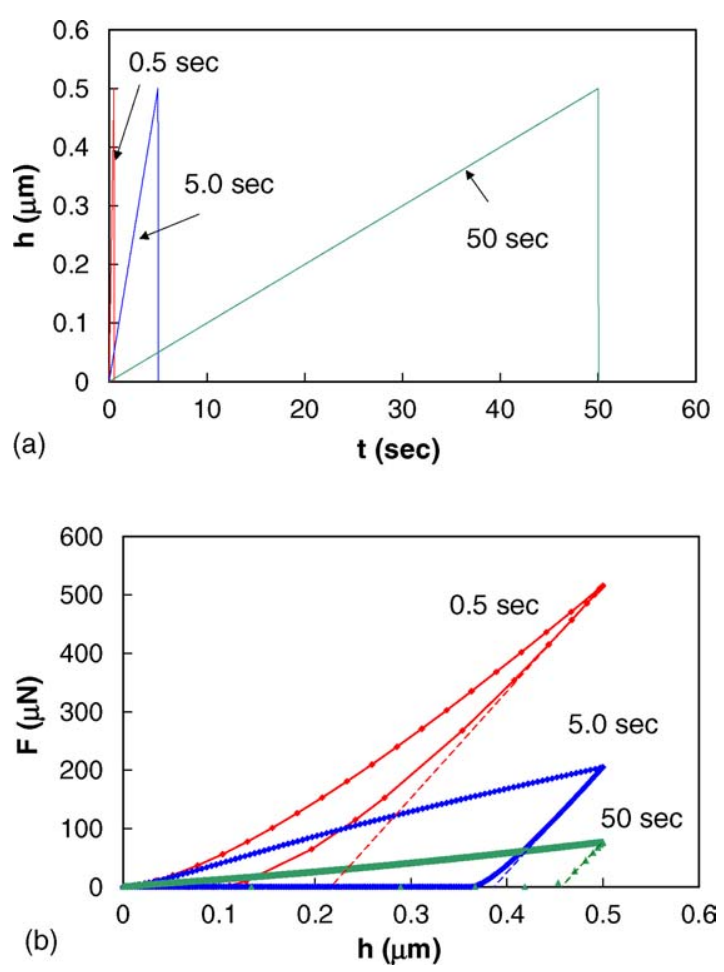

Fig. 4. Displacement-time profiles (a) and the calculated loading-unloading curves (b) for three different loading rates and sufficiently fast unloading rates. The tangent lines with initial unloading slopes are also shown (b). The loading-unloading curves are labeled by the time duration of loading. 
to Eq. (13) or (17), therefore, the unloading slopes are the same, which is evident from Fig. 4b.

On the other hand, Fig. 4b demonstrates that the Oliver-Pharr procedure for estimating the contact depth using Eq. (14) is not applicable to indentation in viscoelastic solids. This can be seen by the fact that Eq. (14) would have predicted very different contact depths, $h_{\mathrm{c}}$, since $F_{\mathrm{m}}$ is different while $h_{\mathrm{m}}$ and $(\mathrm{d} F / \mathrm{d} h)_{\mathrm{m}}$ are the same for the three cases, contradicting the fact that $h_{\mathrm{c}}$ is the same. Indeed, the contact depth calculated from the Oliver-Pharr procedure for the three cases shown in Fig. 4b are 0.287, 0.415, and $0.468 \mu \mathrm{m}$ for the $0.5,5.0$, and $50 \mathrm{~s}$ loading time, respectively. In contrast, the actual contact depth for all three cases is about $0.257 \pm 0.001 \mu \mathrm{m}$ from finite element calculations. The errors are 12,61 , and $82 \%$, respectively. Errors of the same magnitude are also seen from finite element calculations when load, instead of displacement, is the independent variable. We note that the Oliver-Pharr method always overestimates the contact depth, resulting in underestimating the modulus values determined using Eq. (13) or (17). Thus, the Oliver-Pharr procedure may cause significant error in determining contact depth or contact area when it is applied to the analysis of spherical indentation in viscoelastic solids. This observation is not surprising since the Oliver-Pharr procedure, specifically Eq. (14), was derived using Eq. (7), which is only valid for spherical indentation in purely elastic solids.

\section{Conclusions}

Using analytical and finite element modeling, we have investigated spherical indentation in linear viscoelastic solids. We have established a relationship between contact depth and indenter displacement and a relationship between initial unloading slope, contact depth, and the instantaneous modulus for sufficiently high rate of unloading. We have shown that the Oliver-Pharr method for estimating the contact depth, Eq. (14), is not applicable to spherical indentation in viscoelastic solids.

These analytical and numerical results form the basis for performing spherical indentation measurements in linear viscoelastic solids. Specifically, Eqs. (10) and (12) imply that:

(1) Fast unloading is essential in determining the instantaneous modulus from initial unloading slope using Eq. (13) or (17). The numerical examples in this paper suggest that "sufficiently fast" unloading is achieved when the time duration of liner unloading is about $0.1-0.01$ times the relaxation time of linear viscoelastic materials. In practice, the manifestation of sufficiently fast unloading is the "converged" unloading curve that can be obtained using different unloading rates spanning several order of magnitudes. The development and improvement of indentation equipment that allow fast and slow responses are thus encouraged.

(2) A number of authors have suggested adding a "hold-atpeak-load" period between loading and unloading to avoid the occurrence of the "bulge" on the unloading curves [25,26,29-34]. It has been shown recently that the initial unloading slope is nevertheless a function of holding history and unloading condition [29-34]. A correction formula has been proposed by Ngan and coworkers for the initial unloading slope after the hold period so that the instantaneous modulus can be obtained from unloading [31-34]. Eqs. (10) and (12) suggest, however, that the hold-period may be unnecessary if unloading can be made sufficiently fast so that the instantaneous modulus can be obtained from the unloading slope.

(3) For spherical indentation a simple relationship exists between contact depth and indentation depth (Eq. (8) or (15)) from which contact area can be determined without invoking the Oliver-Pharr method.

These general guidelines should help improve the accuracy and reproducibility of spherical indentation measurements for determining properties of purely linear viscoelastic solids.

\section{Acknowledgements}

The authors would like to thank Wangyang Ni, Mike Lukitsch, Yue Qi, Tom Perry, and Wes Capehart, Lou Hector, and Mark W. Verbrugge for valuable discussions. C.-M. Cheng would like to acknowledge partial support from NSF of China, Project No. 10372101.

\section{References}

[1] E.H. Lee, Q. Appl. Math. 13 (1955) 183.

[2] J.R.M. Radok, Q. Appl. Math. 15 (1957) 198.

[3] E.H. Lee, J.R.M. Radok, J. Appl. Mech. 27 (1960) 438.

[4] S.C. Hunter, J. Mech. Phys. Solids 8 (1960) 219.

[5] G.A.C. Graham, Int. J. Eng. Sci. 3 (1965) 27.

[6] G.A.C. Graham, Int. J. Eng. Sci. 5 (1967) 495.

[7] W.H. Yang, J. Appl. Mech. 33 (1966) 395.

[8] T.C.T. Ting, J. Appl. Mech. 33 (1966) 845.

[9] T.C.T. Ting, J. Appl. Mech. 35 (1968) 248.

[10] L. Cheng, X. Xia, W. Yu, L.E. Scriven, W.W. Gerberich, J. Polym. Sci.: Part B: Polym. Phys. 38 (2000) 10.

[11] P.-L. Larrson, S. Carlsson, Polym. Test. 17 (1998) 49.

[12] S. Shimizu, T. Yanagimoto, M. Sakai, J. Mater. Res. 14 (1999) 4075 .

[13] M. Sakai, S. Shimizu, J. Non-Cryst. Solids 282 (2001) 236.

[14] M. Sakai, Philos. Mag. A82 (2002) 1841.

[15] M.L. Oyen, R.F. Cook, J. Mater. Res. 18 (2003) 139.

[16] W.C. Oliver, G.M. Pharr, J. Mater. Res. 7 (1992) 1564.

[17] W.C. Oliver, G.M. Pharr, J. Mater. Res. 19 (2004) 3.

[18] H. Lu, B. Wang, J. Ma, G. Huang, H. Viswanathan, Mech. Time Depend. Mater. 7 (2003) 189.

[19] M.V. Ramesh Kumar, R. Narasimhan, Curr. Sci. 87 (2004) 1088.

[20] Y.-T. Cheng, C.-M. Cheng, Mater. Sci. Eng. Rep.: Rev. J. R44 (2004) 91.

[21] Y.-T. Cheng, C.-M. Cheng, J. Mater. Res. 20 (2005) 1046.

[22] W.N. Findley, J.S. Lai, K. Onaran, Creep Relaxation of Nonlinear Viscoelastic Materials, Dover, Mineola, 1989.

[23] G.T. Mase, G.E. Mase, Continuum Mechanics for Engineers, second ed., CRC, Boca Raton, 1999.

[24] I.N. Sneddon, Int. J. Eng. Sci. 3 (1965) 47.

[25] M.R. VanLandingham, J. Res. Nat. Inst. Stand. Tech. 108 (2003) 249. 
[26] B.J. Briscoe, L. Fiori, E. Pelillo, J. Phys. D: Appl. Phys. 31 (1998) 2395.

[27] HKS, Inc., Pawtucket, Rhode Island 02860, USA.

[28] W. Ni, Y.-T. Cheng, C.-M. Cheng, D.S. Grummon, J. Mater. Res. 19 (2004) 149.

[29] G. Hochstetter, A. Jimenez, J.L. Loubet, J. Macromol. Sci. Phys. B38 (1999) 681.
[30] T. Chudoba, F. Richter, Surf. Coat. Technol. 148 (2001) 191.

[31] G. Feng, A.H.W. Ngan, J. Mater. Res. 17 (2002) 660.

[32] A.H.W. Ngan, B. Tang, J. Mater. Res. 17 (2002) 2604.

[33] B. Tang, A.H.W. Ngan, J. Mater. Res. 18 (2003) 1141.

[34] A.H.W. Ngan, H.T. Wang, B. Tang, K.Y. Sze, Int. J. Solids Struct. 42 (2005) 1831. 